\title{
Gradient-Echo Line Scan Imaging Using 2D-Selective RF Excitation
}

\author{
Jürgen Finsterbusch ${ }^{1}$ and Jens Frahm \\ Biomedizinische NMR Forschungs GmbH am Max-Planck-Institut für Biophysikalische Chemie, 37070 Göttingen, Germany \\ E-mail: jfinste@gwdg.de
}

Received April 4, 2000; revised June 29, 2000

\begin{abstract}
A gradient-echo line scan imaging technique was developed which employs two-dimensional spatially selective radiofrequency (2DRF) pulses for consecutively exciting individual columns of transverse magnetization, i.e., image lines. Although a variety of trajectories are possible for 2DRF excitation, the current implementation involved a blipped-planar trajectory in conjunction with additional saturation RF pulses to suppress side excitations above and below the desired image section, i.e., along the blip direction of the 2DRF pulse. Human brain imaging at 2.0 T (Siemens Vision, E rlangen, Germany) resulted in measuring times of $5.2 \mathrm{~s}$ for a $5-\mathrm{mm}$ section at $1.0 \times 1.0 \mathrm{~mm}$ in-plane resolution. Functional neuroimaging of the motor cortex at $1.2 \mathrm{~s}$ temporal resolution and $0.78 \times 1.56 \mathrm{~mm}$ in-plane resolution exploited the capability of imaging inner volumes (here a $25-\mathrm{mm}$ strip) without signal aliasing. $\bigcirc 2000$ Academic Press
\end{abstract}

Key Words: magnetic resonance imaging; line scan imaging; 2D-selective RF excitation; inner-volume imaging; fMRI.

\section{INTRODUCTION}

Line scan imaging (LSI) relies on consecutive excitations of columnar volumes of transverse magnetization that are readout in the presence of a one-dimensional frequency-encoding gradient. Fourier transformation and subsequent arrangement of pertinent projection "lines" yield a two-dimensional image which does not require phase-encoding and offers imaging of arbitrary fields-of-view (FOV) determined by the number of acquired lines. Thus, in contrast to Fourier imaging, LSI allows imaging of inner volumes without suffering from signal aliasing. Obviously, the technique is insensitive to phase distortions that, for example, occur in the presence of motion and normally propagate through image space.

Extending early approaches in the infancy of MRI (1-5), more recent LSI techniques have attempted to reduce the imaging time $(6,7)$ and even resulted in single-shot sequences (8-10) with applications to cardiac (11) and diffusionweighted imaging $(12,13)$. These techniques define lines of magnetization by cross-sectional multipulse RF excitations

\footnotetext{
${ }^{1}$ To whom correspondence should be addressed.
}

that generate RF refocused echoes of a columnar intersection volume.

This work presents a novel concept for LSI by combining two-dimensional spatially selective RF (2DRF) excitation pulses (14) with a gradient-echo acquisition of the resulting columnar magnetization (GE-LSI). At least in principle, 2DRF GE-LSI allows the isolated excitation of individual image lines. This feature translates into the possibility of covering a three-dimensional volume by parallel but otherwise arbitrarily positioned lines without interference from consecutive acquisitions. Here we describe the theoretical framework for optimizing suitable 2DRF pulses and demonstrate experimental implementations of LSI on a conventional whole-body MRI system at $2.0 \mathrm{~T}$. Because of the acquisition of gradient echoes, GE-LSI measurements may be rapidly repeated by using low flip angles for 2DRF excitation. New fields of application evolve from the inherent $\mathrm{T} 2 *$ contrast. This work demonstrates functional neuroimaging of the human motor cortex by LSI which not only exploits the inherent motion robustness but also allows for a better temporal resolution as conventional gradient-echo imaging by restricting the FOV while maintaining high spatial resolution.

\section{THEORY}

A helpful construct for analyzing data acquisition schemes in MRI is the k-space. In close analogy, Pauly et al. (15) introduced the concept of $\mathbf{K}$-space to describe low flip angle RF excitations in the presence of time-dependent magnetic field gradients $\mathbf{G}(t)$. Accordingly, the trajectory of a spatially selective RF excitation between $t_{0}$ and $t_{1}$,

$$
\mathbf{K}(t)=-\gamma \int_{t}^{t_{1}} \mathbf{G}\left(t^{\prime}\right) d t^{\prime} \quad t_{0} \leq t \leq t_{1},
$$

represents a one-dimensional line in the three-dimensional $\mathbf{K}$-space along which the effective gradient integral "moves" during the evolution of the RF pulse. Assuming thermodynamic equilibrium before excitation with $M_{0}(\mathbf{r})$, the equilibrium magnetization, the dimensionless excitation profile 


$$
P(\mathbf{r})=\frac{M_{\perp}\left(t_{1}, \mathbf{r}\right)}{M_{0}(\mathbf{r})}
$$

defines the relative amount of longitudinal magnetization flipped into the transverse plane, i.e., $M_{\perp}\left(t_{1}, \mathbf{r}\right)$. The profile is given by

$$
P(\mathbf{r})=\mathscr{F}(W(\mathbf{K}) \cdot S(\mathbf{K}))=\mathscr{F}(W(\mathbf{K})) * \mathscr{F}(S(\mathbf{K})),
$$

with $\mathscr{F}$ the Fourier transformation (FT) and $*$ denoting convolution (see the Appendix). The weighting function $W$ describes the $B_{1}$-field along the trajectory as a function of $\mathbf{K}$ instead of $t$. The sampling function $S$ equals 1 for $\mathbf{K}$ values that are part of the trajectory and 0 elsewhere. If $S=1$ for all $\mathbf{K}$, i.e., if the trajectory covers the $\mathbf{K}$-space completely, the resulting excitation profile $P$ is given by the FT of the weighting function $W$. Or conversely, for a desired RF excitation, its FT should be chosen as the weighting function.

In practice, sampling is restricted to a finite region in $\mathbf{K}$ space. As a consequence, the convolution in Eq. [3] causes truncation and blurring of the excitation profile. The effects become more pronounced for smaller sampled regions. Columnar excitation volumes lead to an additional problem as the required weighting function does not vanish on a two-dimensional area in $\mathbf{K}$-space. Because the one-dimensional trajectory $\mathbf{K}(t)$ covers the $\mathbf{K}$-space only along discrete lines, the convolution of the FT of the weighting function with the FT of the sampling function, i.e., a function of discrete lines itself, results in an excitation profile with periodic "side" excitations. Whereas their specific shape depends on the chosen trajectory, their distance increases for higher sampling densities in $\mathbf{K}$ space in accordance with FT properties. For GE-LSI the side excitations pose a problem that needs to be addressed by spatial saturation (see below).

\section{EXPERIMENTAL}

LSI was implemented on a 2.0-T whole-body MRI system (Magnetom Vision, Siemens, Erlangen, Germany) equipped with $25 \mathrm{mT} \mathrm{m}^{-1}$ gradients (maximum slew rate $40 \mathrm{~T} \mathrm{~m}^{-1} \mathrm{~s}^{-1}$ ). All measurements were performed with use of the standard headcoil. Image processing was adapted to the requirements of LSI by replacing the FT and zero filling in the direction of the moving lines by a linear interpolation.

For studies of healthy volunteers, informed written consent was obtained prior to the examination. Functional mapping of the human motor cortex hand area involved a sequential fingerto-thumb opposition task in a protocol comprising six cycles of task performance ( $24 \mathrm{~s}$ ) and motor rest (48 s). The data analysis was based on statistically thresholded maps of correlation coefficients following the ideas outlined in Ref. (16). It employed a box-car reference function shifted by three images (3.6 s) with respect to stimulus onset.

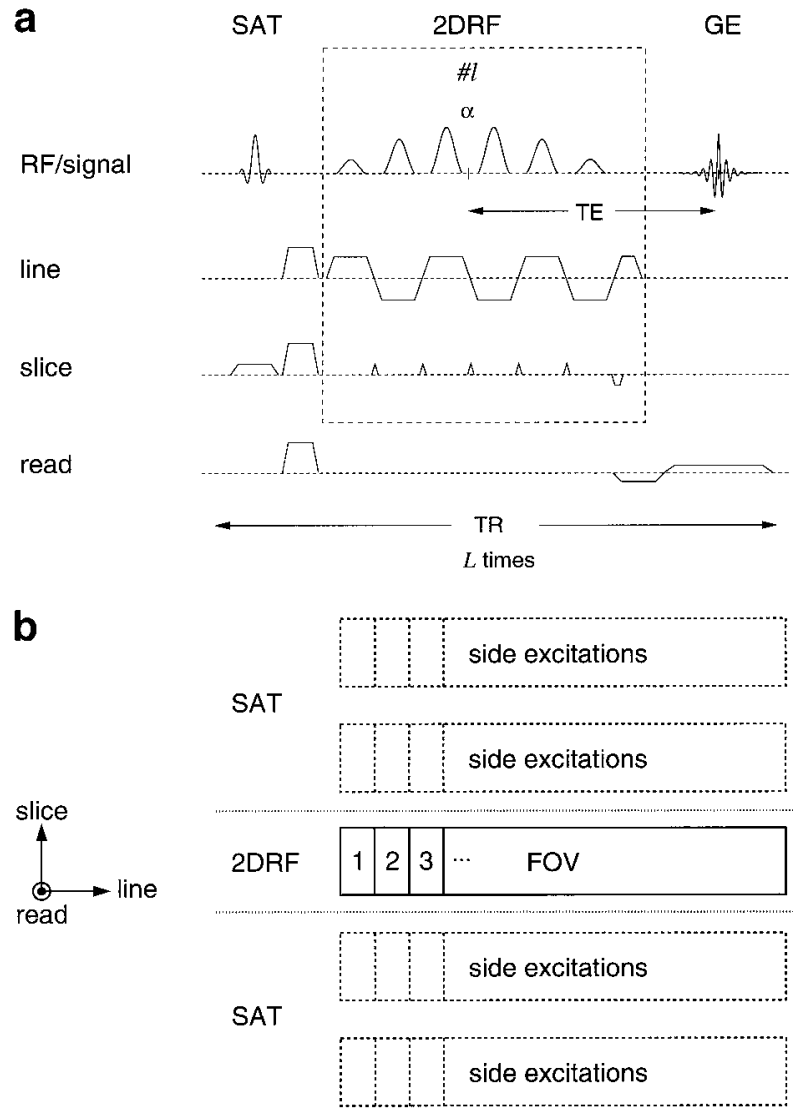

FIG. 1. (a) Basic single-slice sequence for gradient-echo line scan imaging (GE-LSI). 2DRF excitation of a columnar volume is based on a blippedplanar trajectory (six lines) complemented by RF saturation (SAT) to suppress side excitations along the blip direction. Repetition of the sequence (repetition time TR) covers the desired FOV with $L$ lines using spatially shifted 2DRF excitations. (b) Schematic representations of the image section (FOV), 2DRF excitations, side excitations, and RF saturation areas.

\section{Gradient-Echo LSI Using 2DRF Excitation}

Figure 1 shows (a) the single-slice sequence for 2DRF GE-LSI consisting of 2DRF excitation (dashed box), outervolume saturation (SAT), and acquisition of a frequency-encoded gradient echo (GE), as well as (b) a schematic representation of the desired FOV, the individual line-selective excitations, the side excitations, and the RF saturation areas. For 2DRF excitation a blipped-planar trajectory with six lines was used to excite a columnar volume with full widths at half maximum (FWHM) of 1.0-2.0 mm (line) and $5 \mathrm{~mm}$ (section thickness), respectively. The trajectory generated side excitations above and below the image section (dashed boxes in Fig. 1b) which were suppressed with use of a single 2.5-ms RF pulse for two-sided saturation. The respective volume was adapted to the desired application and, for example, covered $128 \mathrm{~mm}$ on either side of the section sparing a central region of $\pm 7 \mathrm{~mm}$. The gradient strength for saturation was decreased from 10 to $0.3 \mathrm{mT} \mathrm{m}^{-1}$ during the central part of the pulse to reduce RF power requirements. Spoiler gradients were applied 
a

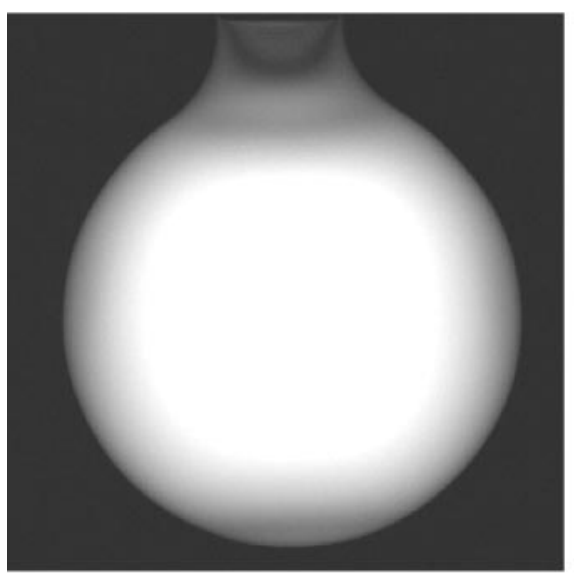

C

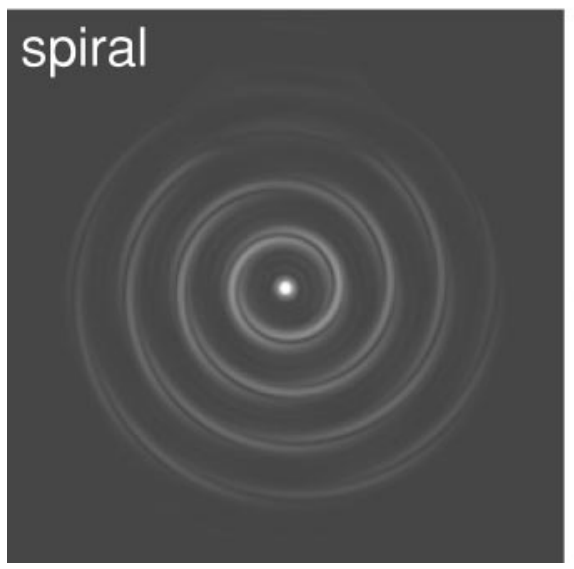

b

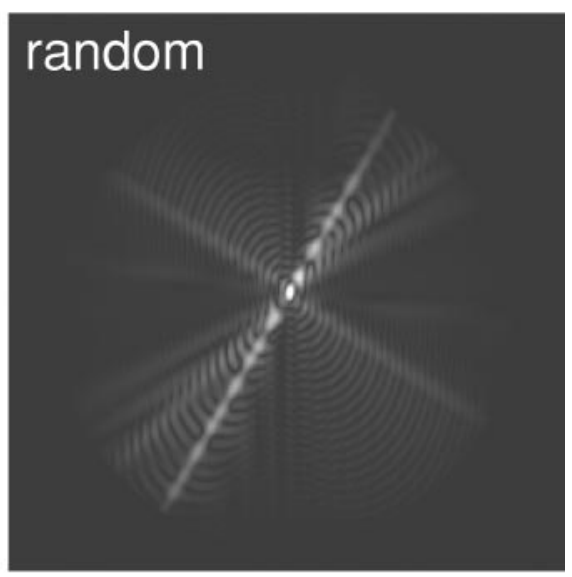

d

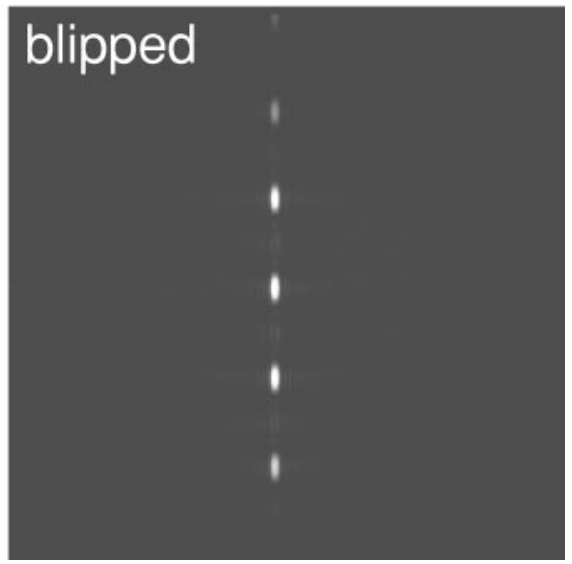

FIG. 2. Magnetization profiles in a water phantom (diameter $160 \mathrm{~mm}$ ) obtained with use of (a) a nonselective RF pulse and (b-d) a 2DRF pulse (12-ms duration, 2-mm horizontal FWHM) with a pseudo-random, spiral, and blipped-planar trajectory, respectively. The lower intensity of the outer side excitations reflects the reduced thickness of the spherical phantom.

in three orthogonal directions with alternating polarities in successive repetitions.

The 2DRF and saturation pulses were calculated using a software package developed by Thomas Kluge for Siemens Medical Systems (17). It determines the RF pulse shape for user-defined gradient trajectories and excitation profiles. All calculated 2DRF envelopes were filtered with a two-dimensional Gaussian function truncated at the $15 \%$ level to compromise between short pulse durations and acceptable truncation effects.

\section{RESULTS AND DISCUSSION}

\section{DRF Excitation}

The choice of a particular 2DRF pulse for GE-LSI depends on imaging features such as the desired T2* contrast, i.e., echo time, and spatial resolution, i.e., section and line thickness.
Thus, the key design parameters are the 2DRF pulse duration, the absolute FWHM of the excited columnar volume, and the nature and extent of side excitations. For anatomic imaging the current implementation aimed at an in-plane resolution of 1-2 $\mathrm{mm}$, a section thickness of $5 \mathrm{~mm}$, and a maximum 2DRF pulse duration of $12-14 \mathrm{~ms}$ to minimize the resulting T2* weighting. Because side excitations strongly depend on the actual K-space sampling, Fig. 2 discusses the problem in more detail as a function of gradient trajectory.

In particular, we evaluated the usefulness of a pseudorandom trajectory (Fig. 2b), a spiral trajectory (Fig. 2c) covering an angular range of $9 \pi$ in $\mathbf{K}$-space, and a blipped-planar trajectory (Fig. 2d) sampling nine parallel lines in K-space. In all cases 2DRF pulses with a duration of $12 \mathrm{~ms}$ and a FWHM of $2 \mathrm{~mm}$ in line direction were employed. This high resolution required a correspondingly large region to be covered in the respective direction of $\mathbf{K}$-space which-for the given pulse duration and gradient slew rates-resulted in a relatively low 
sampling density and therefore caused periodic side excitations.

In principle, the use of a pseudo-random trajectory appears attractive because ideally the side excitations are smeared over the whole object rather than occurring in focused locations "moving" with the 2DRF position. However, for the given hardware, resolution, and pulse duration, the trajectory could not be made sufficiently "random" to avoid side excitations outside a single central spot (Fig. 2b). Nevertheless, for longer pulse durations or lower resolution or, more likely, for faster gradient systems, the pseudo-random approach could become the trajectory of choice.

Spiral trajectories yield a sharp circularly shaped central column of magnetization (Fig. 2c) but suffer from ring-shape excitations that limit the accessible volume in all directions. Under the chosen experimental conditions the first side excitations occur at a radial distance of $20 \mathrm{~mm}$ from the main excitation. Their saturation would interfere with the acquisition of future image lines in arbitrary orientations if the FOV exceeds $15 \mathrm{~mm}$. Within this volume the spiral trajectory results in a two-dimensional section thickness of only $2 \mathrm{~mm}$, whereas a corresponding blipped-planar trajectory would have closer side excitations at a distance of about $12 \mathrm{~mm}$.

The practical advantage of the blipped-planar trajectory (Fig. 2d) is due to the fact that the side excitations are restricted to only one direction, i.e., perpendicular to the sampled lines along the blip direction. Accordingly, the measurable FOV is not limited along the direction of the moving lines. Because RF saturation of the side excitations may also be easily accomplished by standard slice-selective RF pulses above and below the image section, the blipped-planar trajectory lends itself to a practical implementation for single-slice GE-LSI. The repetitive application of saturation pulses together with each lineselective 2DRF pulse maximizes the saturation effect for the acquisition of subsequent lines in a larger FOV.

The MRI system used here suffered from a memory limitation restricting the total number of supporting points for RF pulse definition. This particularly affects the use of a large number of individual line-selective 2DRF pulses if time-dependent gradients require a complex phase modulation. Because all 2DRF pulses must be stored before starting the LSI acquisition, the corresponding memory buffer was filled for as few as 10 image lines. For the present implementation of the blipped-planar trajectory this limitation was circumvented by applying RF power only during periods of constant gradient strength. Thus, the spatial line shift was accomplished by adding a frequency offset to a single stored 2DRF pulse. Although feasible, this solution was at the expense of a considerable loss in performance or efficiency of $\mathbf{K}$-space coverage. To avoid the otherwise necessary prolongation of the 2DRF pulse, the sampling density was reduced from 9 to 6 $\mathbf{K}$-space lines, which resulted in closer side excitations. a
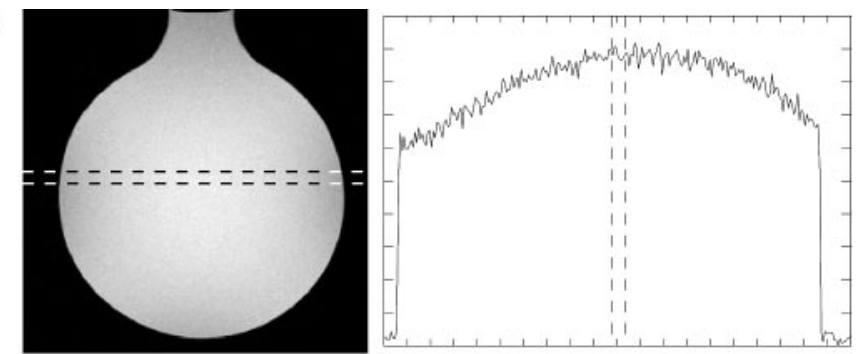

b
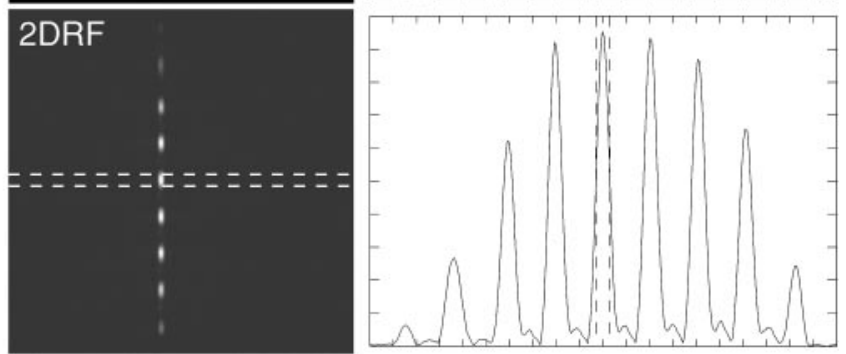

C
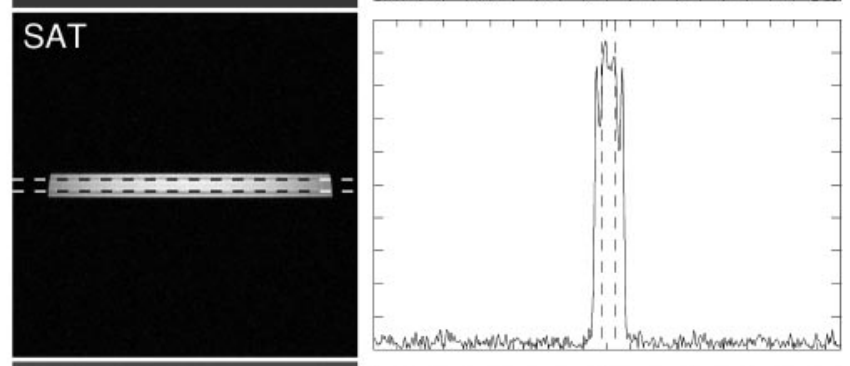

d $\mathrm{SAT}+2 \mathrm{DRF}$
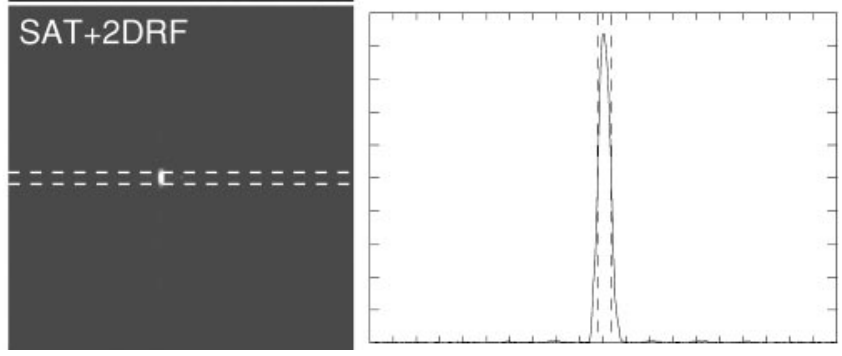

FIG. 3. Magnetization profiles (left) for GE-LSI of a coronal section (dashed box) and perpendicular section profiles (right) obtained with use of (a) a slice-selective RF pulse, (b) a 2DRF pulse, (c) a slice-selective RF and saturation pulse, and (d) a 2DRF and saturation pulse.

\section{Gradient-Echo LSI Using 2DRF Excitation}

Figure 3 demonstrates the experimental results for a GE-LSI sequence using a blipped-planar trajectory with six lines for 2DRF excitation. The target section is a coronal plane through a water phantom, as indicated by a dashed box. The individual magnetization profiles visualize the excitation properties of the 2DRF and saturation pulses. Using a conventional slice-selective excitation pulse (Fig. 3a, left) the signal intensity profile perpendicular to the target section reflects the RF inhomogeneity of the headcoil (right). Application of the 2DRF pulse (Fig. 3b) restricts the excitation in two dimensions by exciting a central strip (line) of magnetization, i.e., a projection along the third dimension, with a 5-mm section thickness and a 2-mm line thickness. However, the total profile also documents sig- 
a
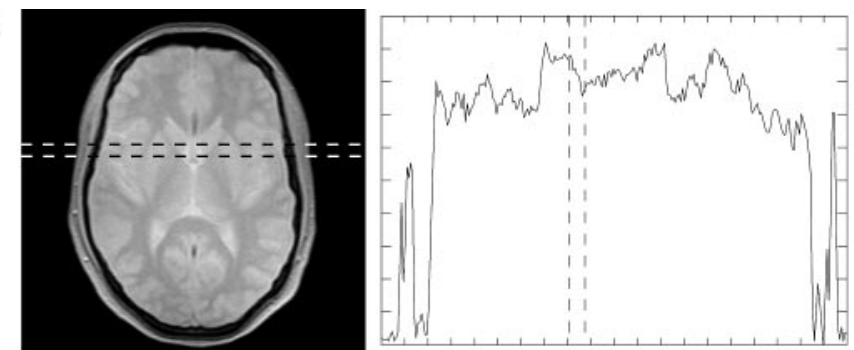

b
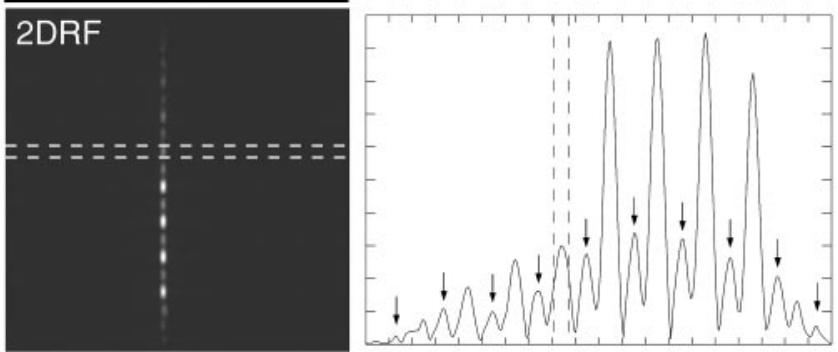

\section{C}

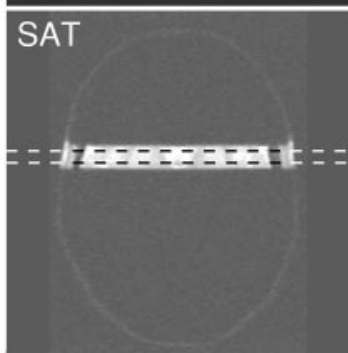

d
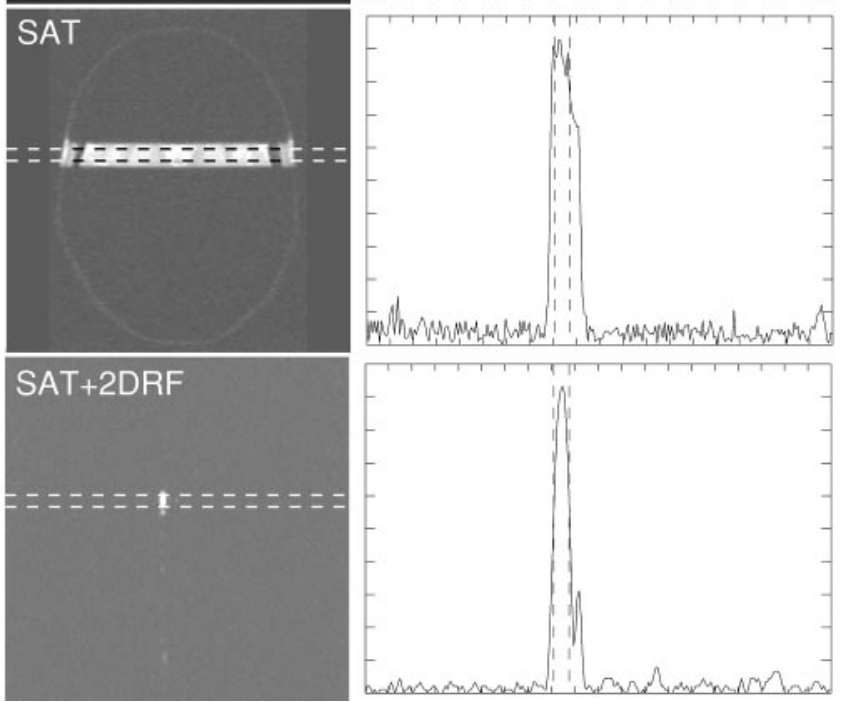

FIG. 4. Magnetization profiles (left) and section profiles (right) as shown in Fig. 3 for application to the human brain. (a) Slice-selective RF pulse, (b) 2DRF pulse, (c) slice-selective RF and saturation pulse, and (d) 2DRF and saturation pulse. In contrast to phantom studies, $2 \mathrm{DRF}$ pulses not only lead to periodic side excitations but also cause $N / 2$ ghosts (arrows).

nificant contributions from side excitations as discussed in the previous section. Together with a two-sided RF saturation pulse (Fig. 3c), the combined profile achieves the desired projection quality of a single excited line of magnetization (Fig. 3d).

In close analogy to the phantom study, Fig. 4 depicts corresponding results for an application of the same GE-LSI sequence to the human brain. Because of the heterogeneous structure of the brain, some of the 2DRF side excitations exceed the intensity of the central line. In particular, the high posterior intensities may be explained by the fact that the underlying coronal projections not only cover the cerebrum but also include parts of the cerebellum, midbrain, and spine, whereas anterior projections are reduced by air cavities. More- over, in contrast to the phantom studies, the 2DRF excitation profile reveals additional excitations (arrows) which occur in between the periodic side excitations from the blip gradient. They are denoted as N/2 excitations because they originatesimilar to the N/2 ghosts in echo-planar imaging with blippedplanar trajectories-from slightly imbalanced gradients when traversing the $\mathbf{K}$-space in opposite directions. In phantoms pertinent effects can be minimized by fine-tuning the timing of the 2DRF pulse relative to that of the underlying gradients. Under in vivo conditions, such efforts neither seem justifiable nor were they entirely successful. In fact, because the time shift for a minimal N/2 excitation was found to be dependent on the location of the selected line reflecting spatially inhomogeneous gradients, respective adjustments would have to be performed for each line individually. For GE-LSI, all side and N/2 excitations had to be removed by RF saturation (Fig. 4c).

Unfortunately, for the present implementation the first $\mathrm{N} / 2$ excitation occurred rather close to the image section. An acceptable suppression of side and N/2 excitations could be achieved by a combination of 2DRF excitation and RF saturation, although the resulting profile (Fig. 4d) was contaminated by a residual N/2 excitation and some tissue-dependent signal loss from the tightly adjusted saturation pulse. The signal attenuation ranged from about $10 \%$ for cerebrospinal fluid to $40 \%$ for white matter and may be explained by the individual water resonance frequency distribution. It is much broader in white matter than in CSF, yielding a more pronounced overlap with the saturation profile. It should be emphasized, however, that this signal loss is not a general problem but is caused by the aforementioned memory limitations. The current generation of MRI systems offers more flexible sequence programming as well as greater gradient slew rates, so that the distance of both side and N/2 excitations may be considerably increased. In addition, the use of stronger gradients will improve the profile for spatially selective saturation.

\section{Anatomic and Functional Neuroimaging}

Table 1 summarizes the experimental parameters used here for 2DRF GE-LSI. The two approaches tested were anatomic imaging of the human brain and functional mapping of a focused area emphasizing high temporal resolution without sacrificing high spatial resolution in a restricted FOV.

Figure 5 shows coronal brain sections of $5-\mathrm{mm}$ thickness obtained at an in-plane resolution of $1.0 \times 2.0 \mathrm{~mm}$ and $1.0 \times$ $1.0 \mathrm{~mm}$. The images were acquired within measuring times of 2.8 and $5.2 \mathrm{~s}$, respectively, which are similar to those for conventional gradient-echo MRI sequences. Interestingly, however, the basic contrast mainly reflects spin density differences despite the use of $90^{\circ}$ flip angles. This result is due to the fact that each line is excited only once during image acquisition and therefore stems from unsaturated longitudinal magnetization. As a consequence, CSF leads to the highest image intensities and gray matter appears brighter than white matter. 
TABLE 1

Parameters for GE-LSI U sing 2DRF Excitation

TA (s)

Resolution (mm)

Interpolation $(\mathrm{mm})$

FOV (mm)

$T_{2 \mathrm{DRF}}(\mathrm{ms})$

Flip angle

TE (ms)

$T_{\text {acq }}(\mathrm{ms})$

$\mathrm{BW}(\mathrm{Hz})$

TR (ms)
2.8

$1.0 \times 2.0$
$1.0 \times 1.0$
$120 \times 256$

10.3

$90^{\circ}$

14.2

12.3

81

40
5.2

$1.0 \times 1.0$

$120 \times 256$

$\begin{array}{cc}14.2 & 11.6 \\ 90^{\circ} & 50^{\circ} \\ 14.2 & 30.0 \\ 8.5 & 30.7 \\ 118 & 33 \\ 40 & 75\end{array}$

Note. TA, total acquisition time; FOV, field-of-view; $T_{2 \mathrm{DRF}}$, duration of the 2DRF pulse; TE, echo time; $T_{\text {acq }}$, acquisition time per echo; BW, bandwidth per pixel; TR, line repetition time.

Additional $\mathrm{T} 2 *$ sensitivity emerges from the timing of the gradient echo. In this respect, the achievable echo time exceeds that of comparable FLASH sequences as the duration of the 2DRF pulse (10-14 ms) exceeds that of a typical slice-selective RF pulse (1-3 ms). Also, the repetition time of GE-LSI sequences is slightly longer to include the RF saturation pulse and accompanying spoiler gradients $(10 \mathrm{~ms})$. However, this penalty in imaging time may be alleviated by the ability to focus on the actual FOV without problems due to (phaseencoded) undersampling and related signal aliasing (see below). Although the signal-to-noise ratio (SNR) is reduced by a factor of the square root of the number of lines compared to Fourier imaging, some compensation is achieved by the use of full $90^{\circ}$ excitations. This particularly applies if spin density contrast is a desired feature.

Functional mapping of brain activation may be a potential niche application of GE-LSI because it benefits from the absence of T1 contrast (i.e., motion and flow sensitivity) and has $\mathrm{T} 2 *$ sensitivity (i.e., functional deoxyhemoglobin contrast). GE-LSI sequences may also offer a good compromise with respect to spatiotemporal resolution when applied to specific brain regions, as in functional studies. Figure 6 shows the results of a study employing a right-hand sequential finger-tothumb opposition task in a block design. Dynamic acquisitions were based on $\mathrm{T} 2 *$-weighted line scan images at an echo time of $30 \mathrm{~ms}$ (Fig. 6a) which covered the left hemispheric motor cortex by an oblique $25-\mathrm{mm}$ FOV at an interpolated resolution of $0.78 \times 0.78 \mathrm{~mm}$ within a measuring time of $1.2 \mathrm{~s}$. Because of the repetitive nature of the mapping experiment, the flip angle was reduced to $50^{\circ}$. The statistically thresholded activation map (Fig. 6b) is overlaid on an averaged line scan image with a $150 \times 200 \mathrm{~mm}$ FOV to better visualize the hand area of the motor cortex. The signal intensity time course from all pixels within the activated area (Fig. 6c) reveals typical blood oxygenation level dependent MRI responses of 3-4\% during task performance.

\section{CONCLUSION}

This work presents the theoretical framework as well as experimental applications for a novel approach to line scan imaging which combines a 2DRF excitation of columnar volumes of magnetization with the acquisition of a frequencyencoded gradient echo. The technique allows rapid imaging of arbitrarily shaped inner volumes without phase-aliasing hampering Fourier imaging. Extending previous LSI methods which are based on cross-sectional multipulse excitations, an ideal 2DRF excitation avoids saturation of neighboring volumes. In practice, however, the use of 2DRF pulses with a reasonable duration causes side excitations which must be removed by spatial saturation. The present implementation was further compromised by a memory limitation of the MRI system which led to trajectories with particularly close side excitations. Current hardware and software improvements are expected to alleviate this aspect of GE-LSI leaving reduced
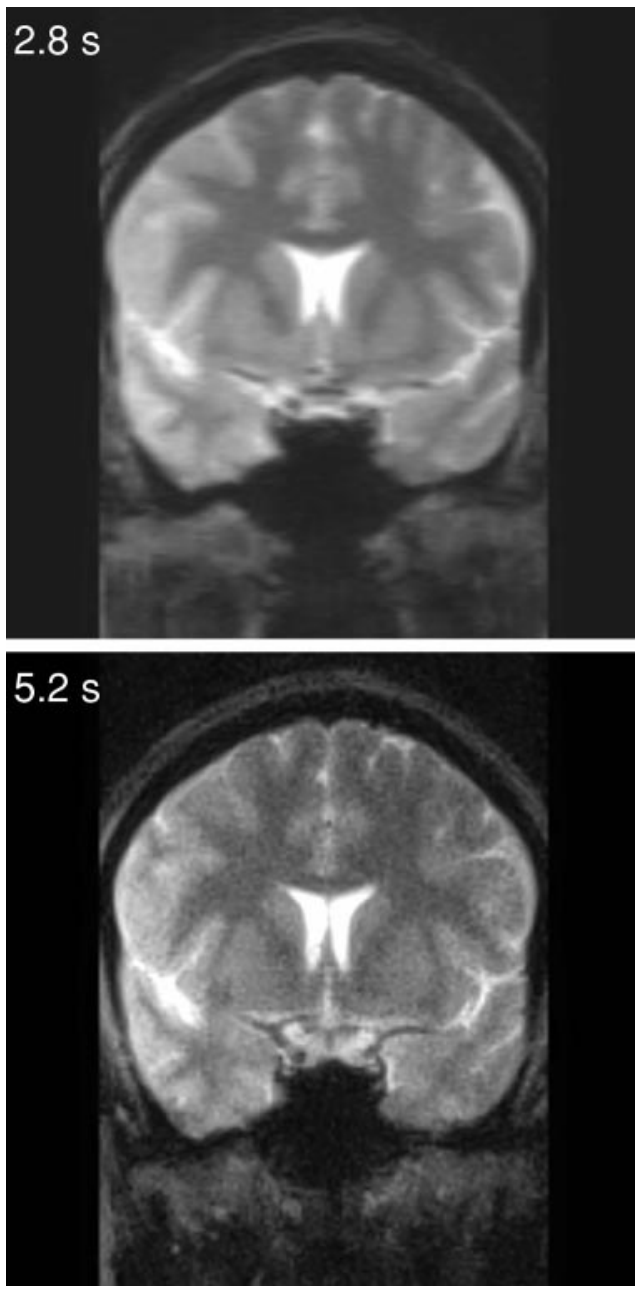

FIG. 5. Coronal GE-LSI of the human brain obtained within $2.8 \mathrm{~s}$ at $1.0 \times$ $2.0 \mathrm{~mm}$ resolution and $5.2 \mathrm{~s}$ at $1.0 \times 1.0 \mathrm{~mm}$ resolution, respectively. The section thickness was $5 \mathrm{~mm}$; for other parameters see Table 1 . 
a

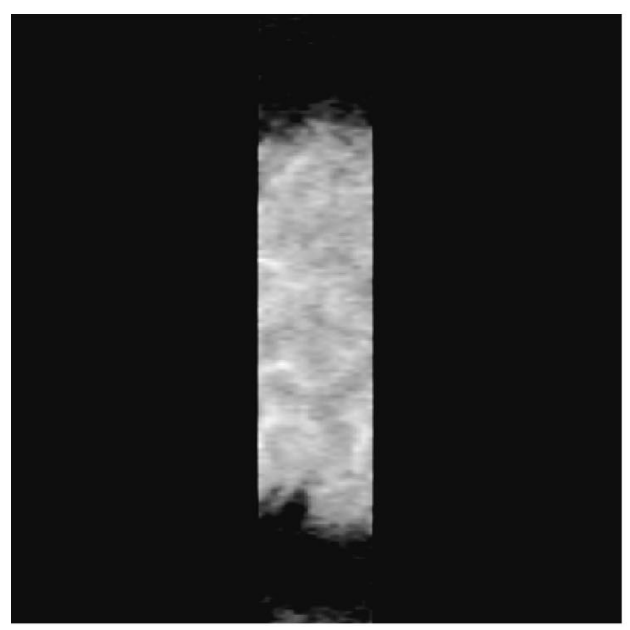

b

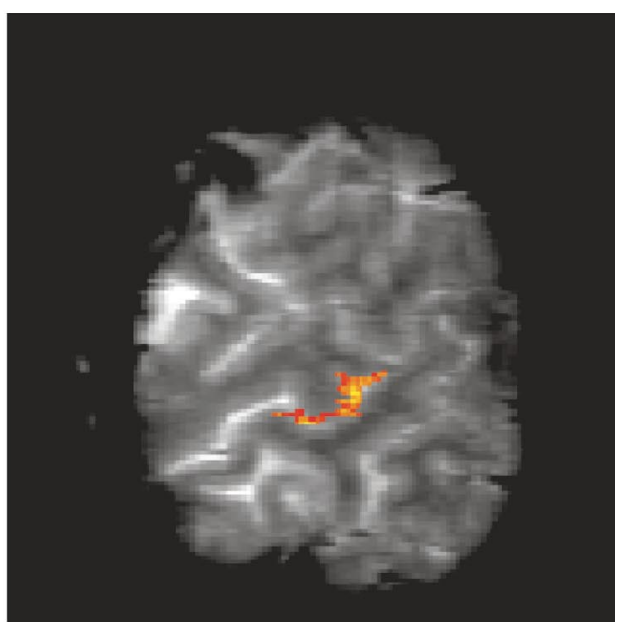

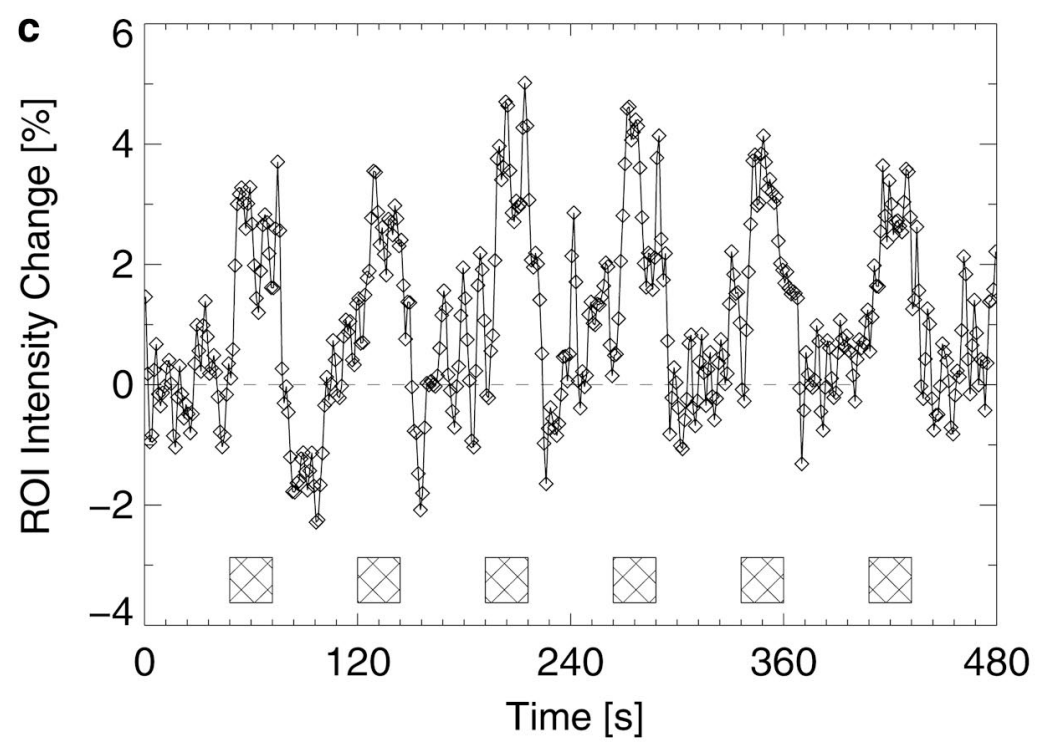

FIG. 6. Functional mapping of the human motor cortex by GE-LSI. (a) Line scan image of a 25 -mm FOV obtained within $1.2 \mathrm{~s}$ at $0.78 \times 1.56 \mathrm{~mm}$ (interpolated to $0.78 \times 0.78 \mathrm{~mm}$ ) resolution and 5 -mm section thickness. The flip angle was $50^{\circ}$; for other parameters see Table 1. (b) Activation map (sequential finger-to-thumb opposition) and (c) corresponding signal intensity time course.

SNR compared to Fourier imaging the main drawback. Apart from anatomic and functional neuroimaging as demonstrated here, GE-LSI may find applications in abdominal MRI, often affected by motion and signal aliasing for small FOVs. Finally, new horizons may emerge from imaging along curved sections and real-time monitoring of interventional procedures.

\section{APPE NDIX}

To simplify the calculations, relaxation effects are ignored. Starting with thermodynamic equilibrium, i.e., $M_{z}=M_{0}$ and $M_{\perp}=0$ at $t=t_{0}$ before RF excitation, the effective $\mathbf{B}$-field (rotating frame) in the presence of time-dependent RF and gradient fields, i.e., $\mathbf{B}_{1}(t)$ and $\mathbf{G}(t)$, is given by

$$
\mathbf{B}_{\text {eff }}(t)=\mathbf{B}_{1}(t)+\mathbf{G}(t) \mathbf{r}
$$

For the complex transverse magnetization

$$
M_{\perp}=M_{x^{\prime}}+i M_{y^{\prime}}
$$

and the complex RF field

$$
B_{1}(t)=B_{1 x^{\prime}}(t)+i B_{1 y^{\prime}}(t)
$$

the equation of motion

$$
\frac{d}{d t} \mathbf{M}=\gamma \mathbf{M} \times \mathbf{B}_{\text {eff }}
$$


transforms to the differential equation

$$
\frac{d}{d t} M_{\perp}(t, \mathbf{r})=-i \gamma \mathbf{G}(t) \mathbf{r} M_{\perp}(t, \mathbf{r})+i \gamma B_{1}(t) M_{z}(t, \mathbf{r}) .
$$

[A5]

In general, an analytic solution of Eq. [A5] is not possible. However, in cases where the longitudinal magnetization is only slightly disturbed by RF excitation (low flip angle approximation), $M_{z}$ can be approximated as constant

$$
M_{z}(t, \mathbf{r}) \approx M_{z}\left(t_{0}, \mathbf{r}\right)=M_{0}(\mathbf{r}) .
$$

Then Eq. [A5] yields

$$
\frac{d}{d t} M_{\perp}(t, \mathbf{r})=-i \gamma \mathbf{G}(t) \mathbf{r} M_{\perp}(t, \mathbf{r})+i \gamma B_{1}(t) M_{0}(\mathbf{r})
$$

and the solution for $t=t_{1}$ (end of RF excitation) is given by

$$
M_{\perp}\left(t_{1}, \mathbf{r}\right)=i \gamma M_{0}(\mathbf{r}) \int_{t_{0}}^{t_{1}} B_{1}(t) \exp \left(-i \gamma \mathbf{r} \int_{t}^{t_{1}} \mathbf{G}\left(t^{\prime}\right) d t^{\prime}\right) d t
$$

Defining

$$
\mathbf{K}(t)=-\gamma \int_{t}^{t_{1}} \mathbf{G}\left(t^{\prime}\right) d t^{\prime} \quad t_{0} \leq t \leq t_{1},
$$

Eq. [A8] can be transformed into

$$
M_{\perp}\left(t_{1}, \mathbf{r}\right)=i \gamma M_{0}(\mathbf{r}) \int_{t_{0}}^{t_{1}} B_{1}(t) e^{i \mathbf{K}(t) \mathbf{r}} d t
$$

$\mathbf{K}(t)$ is termed a trajectory because it represents a one-dimensional line in the three-dimensional $\mathbf{K}$-space which is determined by the values of the gradient integral during RF excitation. Equation [A10] describes the spatial dependency of the transverse magnetization generated by the RF excitation. Although derived under the assumption of low flip angles, it has been shown to be in good agreement with experiments for flip angles as large as $90^{\circ}(15)$.

The following steps replace the dependencies of $t$ by those of $\mathbf{K}$. Using the identities

$$
e^{i \mathbf{K}(t) \mathbf{r}}=\int_{\mathbf{K}} \delta(\mathbf{K}(t)-\mathbf{K}) e^{i \mathbf{K r}} d^{3} \mathbf{K}
$$

and, assuming $\dot{\mathbf{K}}(t) \neq 0$,

$$
1=\frac{|\dot{\mathbf{K}}(t)|}{|\dot{\mathbf{K}}(t)|}
$$

in Eq. [A10] yields

$$
\begin{aligned}
M_{\perp}\left(t_{1}, \mathbf{r}\right)= & i \gamma M_{0}(\mathbf{r}) \int_{t_{0}}^{t_{1}} \int_{\mathbf{K}} B_{1}(t) \frac{|\dot{\mathbf{K}}(t)|}{|\dot{\mathbf{K}}(t)|} \\
& \times \delta(\mathbf{K}(t)-\mathbf{K}) e^{i \mathbf{K r}} d^{3} \mathbf{K} d t .
\end{aligned}
$$

We now introduce the weighting function according to

$$
W(\mathbf{K}(t))=\left.i \gamma \frac{B_{1}(t)}{|\dot{\mathbf{K}}(t)|}\right|_{t=t(\mathbf{K})}
$$

It describes the $B_{1}$-field as a function of $\mathbf{K}$. To ensure uniqueness of $W(\mathbf{K}(t))$, it must be assumed that $\mathbf{K}=\mathbf{K}(t)$ is fulfilled for only one point in time which effectively excludes crossing of the trajectory. With

$$
W(\mathbf{K}(t)) \delta(\mathbf{K}(t)-\mathbf{K})=W(\mathbf{K}) \delta(\mathbf{K}(t)-\mathbf{K})
$$

and interchange of the integration order, Eq. [A13] can be transformed into

$$
\begin{aligned}
M_{\perp}\left(t_{1}, \mathbf{r}\right)= & M_{0}(\mathbf{r}) \int_{\mathbf{K}} \int_{t_{0}}^{t_{1}} \\
& \times W(\mathbf{K})|\dot{\mathbf{K}}(t)| \delta(\mathbf{K}(t)-\mathbf{K}) e^{i \mathbf{K r}} d t d^{3} \mathbf{K} .
\end{aligned}
$$

Equation [A16] contains the definition of the sampling function

$$
S(\mathbf{K})=\int_{t_{0}}^{t_{1}}|\dot{\mathbf{K}}(t)| \delta(\mathbf{K}(t)-\mathbf{K}) d t
$$

For the definition of $S(\mathbf{K})$ the same assumption concerning uniqueness is required as for $W(\mathbf{K}(t))$. In other words, $S(\mathbf{K})=$ 1 for all $\mathbf{K}=\mathbf{K}(t)$ and vanishes otherwise. It thereby identifies those values of $\mathbf{K}$ which are part of the trajectory. Inserting $S(\mathbf{K})$ into Eq. [A16] yields

$$
M_{\perp}\left(t_{1}, \mathbf{r}\right)=M_{0}(\mathbf{r}) \int_{\mathbf{K}} W(\mathbf{K}) S(\mathbf{K}) e^{i \mathbf{K r}} d^{3} \mathbf{K}
$$

or

$$
M_{\perp}\left(t_{1}, \mathbf{r}\right)=M_{0}(\mathbf{r}) \mathscr{F}(W(\mathbf{K}) S(\mathbf{K}))
$$


with $\mathscr{F}$ the Fourier transformation. Using the dimensionless excitation profile

$$
P(\mathbf{r})=\frac{M_{\perp}\left(t_{1}, \mathbf{r}\right)}{M_{0}(\mathbf{r})}
$$

Eq. [A19] is equivalent to

$$
P(\mathbf{r})=\mathscr{F}(W(\mathbf{K})) * \mathscr{F}(S(\mathbf{K}))
$$

with $*$ denoting convolution. Thus, the excited transverse magnetization $M_{\perp}\left(t_{1}, \mathbf{r}\right)$ is given by the convolution of the FT of the weighting function $W(\mathbf{K})$ with the FT of the sampling function $S(\mathbf{K})$.

\section{ACKN OWLEDGMENTS}

We thank Thomas Kluge and Siemens Medical Systems for the software package for calculating RF excitations in the presence of time-dependent magnetic gradient fields.

\section{REFERENCES}

1. P. Mansfield and A. A. Maudsley, Medical imaging by NMR, Br. J . Radiol. 50, 188-194 (1977).

2. P. Mansfield, I. L. Pykett, P. G. Morris, and R. E. Coupland, Human whole body line-scan imaging by NMR, Br. J. Radiol. 51, 921-922 (1978).

3. P. Mansfield, P. G. Morris, R. J . Ordidge, I. L. Pykett, V. Bangert, and R. E. Coupland, Human whole body imaging and detection of breast tumours by NMR, Philos. Trans. R. Soc. Lond. B Biol. Sci. 289, 503-510 (1980).

4. L. E. Crooks, Selective irradiation line scanning techniques for NMR imaging, IEEE Trans. Nucl. Sci. 27, 1239-1244 (1980).

5. A. A. Maudsley, Multiple-line-scanning spin density imaging, J . Magn. Reson. 41, 112-126 (1980).
6. T. A. Case, K. Ganesan, and D. C. Ailion, A real-time roving-linescan planar imaging technique with spurious echo spoiling, in "Proceedings of the Society of Magnetic Resonance in Medicine," 6th Annual Meeting, p. 227, New York, 1987.

7. D. C. Ailion, K. Ganesan, T. A. Case, and R. A. Christman, Rapid line scan technique for artifact-free images of moving objects, Magn. Reson. Imaging 10, 747-754 (1992).

8. D. Jensen and D. Holz, SLIM-A novel approach to fast MR imaging, in "Proceedings of the Society of Magnetic Resonance in Medicine," 7th Annual Meeting, p. 117, San Francisco, 1988.

9. M. E. Meyerand and E. C. Wong, A time encoding method for single-shot imaging, Magn. Reson. Med. 34, 618- 622 (1995).

10. J. Finsterbusch and J. Frahm, Single-shot line scan imaging using stimulated echoes, J. Magn. Reson. 137, 144-153 (1999).

11. M. E. Meyerand, C. H. Moritz, and E. C. Wong, Single-shot, motion insensitive cardiac imaging on a standard clinical system, Magn. Reson. Med. 40, 930-933 (1998).

12. H. Gudbjartsson, S. E. Maier, R. V. Mulkern, I. Á. Mórocz, S. Patz, and F. A. J olesz, Line scan diffusion imaging, Magn. Reson. Med. 36, 509-519 (1996).

13. J. Finsterbusch and J. Frahm, Diffusion-weighted single-shot line scan imaging of the human brain, Magn. Reson. Med. 42, 772-778 (1999).

14. P. A. Bottomley and C. J. Hardy, Two-dimensional spatially selective spin inversion and spin-echo refocusing with a single nuclear magnetic resonance pulse, J. Appl. Phys. 62, 42844290 (1987)

15. J. Pauly, D. Nishimura, and A. Macovski, A k-space analysis of small-tip-angle excitation, J. Magn. Reson. 81, 43-56 (1989).

16. A. Kleinschmidt, M. Requardt, K. D. Merboldt, and J . Frahm, On the use of temporal correlation coefficients for magnetic resonance mapping of functional brain activation: Individualized thresholds and spatial response delineation, Intern. J . Imaging Sys. Technol. 6, 238-244 (1995).

17. T. Kluge, "Zweidimensional ortsselektive Anregung eines Spinensembles mit einem kommerziellen Kernspintomographen," Diploma Thesis, University of Bayreuth, Germany, 1997. 